\title{
Weak Arithmetics and Kripke Models ${ }^{1}$
}

\author{
Morteza Moniri \\ Institute for Studies in Theoretical Physics and Mathematics \\ P.O. Box 19395-5746, Tehran, Iran \\ email: ezmoniri@ipm.ir
}

\begin{abstract}
In the first section of this paper we show that $i \Pi_{1} \equiv W \neg \neg l \Pi_{1}$. In the second section of the paper, we show that for equivalence of forcing and satisfaction of $\Pi_{m}$-formulas in a linear Kripke model deciding $\Delta_{0}$-formulas, it is necessary and sufficient that the model be $\Sigma_{m}$-elementary. This implies that if a linear Kripke model forces $P E M_{\text {prenex }}$, then it forces $P E M$. We also show that, for each $n \geqslant 1$, $i \Phi_{n}$ does not prove $\mathcal{H}\left(I \Pi_{n}\right)$. Here, $\Phi_{n}$ 's are Burr's fragments of $H A$.
\end{abstract}

2000 Mathematics Subject Classification: 03F30, 03F55, $03 \mathrm{H} 15$.

Key words and phrases: Fragments of Heyting Arithmetic, Kripke models

\section{Preliminaries}

We fix the language $L=\{+, \cdot,<, 0,1\}$. The principle $P E M$ (some of whose restrictions will appear below) of Excluded Middle is $\forall \bar{x}(\varphi(\bar{x}) \vee \neg \varphi(\bar{x}))$.

Heything arithmetic $H A$ and its fragments $\left(P A^{-}\right)^{i}$, iop, lop, $i \Delta_{0}, i \Sigma_{n}$ and $i \Pi_{n}, n \geq 1$, are the intuitionistic counterparts of first order Peano Arithmetic $P A$ and its fragments $P A^{-}$, Iop, Lop, $I \Delta_{0}, I \Sigma_{n}$ and $I \Pi_{n}$. More generally for any set $\Gamma$ of formulas we will use notations such as $i \Gamma$ and $l \Gamma$ in the same manner. $\neg \Gamma$ is the class of formulas of the form $\neg \varphi$ with $\varphi \in \Gamma$.

By $W \neg \neg L N P$, we mean the scheme $\forall \bar{y} \neg \neg(\exists x \varphi(x, \bar{y}) \rightarrow \exists x(\varphi(x, \bar{y}) \wedge \forall z<x \neg \varphi(z, \bar{y})))$.

We use the usual terminology about Kripke structures as in [TD]. Here we mention two facts about Kripke models. The proofs are straightforward (see $[\mathrm{AM}]$ ).

Fact 0.1 Suppose $\alpha$ is a node of a Kripke model and $\varphi$ is an $L_{\alpha}$-sentence:

1) $\alpha \Vdash \varphi$ iff $\beta \Vdash \varphi$ for each $\beta \geq \alpha$.

\footnotetext{
${ }^{1}$ This version corrects an error in the journal version.
} 
2) $\alpha \Vdash \neg \varphi$ iff $\beta \nVdash \varphi$ for each $\beta \geq \alpha$.

3) $\alpha \Vdash \neg \neg \varphi$ iff for each $\beta \geq \alpha$ there exists $\gamma \geq \beta$ such that $\gamma \Vdash \varphi$.

Fact 0.2 Suppose $\mathcal{K} \Vdash\left(P A^{-}\right)^{i}$ (resp. $\left.\mathcal{K} \Vdash i \Delta_{0}\right)$ and $\varphi \in \exists_{1}\left(\right.$ resp. $\left.\varphi \in \Sigma_{1}\right)$. Then for each $\alpha \in K$, we have:

$$
\alpha \Vdash \varphi \Leftrightarrow M_{\alpha} \vDash \varphi \text {. }
$$

If $\psi \in \forall_{1}$ (resp. $\psi \in \Pi_{1}$ ) then:

$$
\alpha \Vdash \psi \Leftrightarrow \forall \beta \geq \alpha M_{\beta} \vDash \psi
$$

Therefore, a $\forall_{1}$ (resp. $\Pi_{1}$ )-formula is forced at a node $\alpha$ of a Kripke model of $\left(P A^{-}\right)^{i}$ (resp. $i \Delta_{0}$ ) if and only if it is satisfied in the union of the worlds in any path above $\alpha$.

\section{1. $i \Pi_{1}$ and its Kripke models}

It was observed in [MM, Sec. 6] that, the second proof in [TD, p.131] for $H A \vdash$ $W \neg \neg L N P$ actually proves the following:

Fact 1.1 If a fragment $i \Gamma$ of $H A$ is $m$-closed under the negative translation and $I \Gamma \vdash L \Gamma$, then for any formula $\varphi(x, \bar{y}) \in \Gamma, i \Gamma \vdash \forall \bar{y} \neg \neg(\exists x \varphi(x, \bar{y}) \rightarrow \exists x(\varphi(x, \bar{y}) \wedge \forall z<$ $x \neg \varphi(z, \bar{y})))$.

As a corollary, it was proved that iop $\equiv W \neg \neg l o p$ where $W \neg \neg l o p$ is the intuitionistic theory axiomatized by $\left(P A^{-}\right)^{i}$ plus $W \neg \neg L N P$ on open formulas. Here we prove a similar result for $i \Pi_{1}$.

Note that by the above fact $i \Pi_{1} \vdash W \neg \neg l \Pi_{1}$. Also, using $i \Pi_{1} \equiv i \neg \Pi_{1}$, see [W2, Cor. 6], we have $i \Pi_{1} \vdash W \neg \neg l \neg \Pi_{1}$ where $W \neg \neg l \neg \Pi_{1}$ is the intuitionistic theory axiomatized by $i \Delta_{0}$ plus $W \neg \neg L N P$ on $\neg \Pi_{1}$ formulas.

Proposition $1.2 W \neg \neg l \neg \Pi_{1} \vdash i \Pi_{1}$.

Proof Assume $\mathcal{K} \Vdash W \neg \neg l \neg \Pi_{1}$. Let $\alpha \in \mathcal{K}$ does not force $I_{x} \varphi(x, \bar{y})$, for some $\Pi_{1^{-}}$ formula $\varphi$. Therefore, by the above facts, there will exist a node $\gamma \geqslant \alpha$ with $a, \bar{b} \in M_{\gamma}(\bar{b}$ of the same arity as $\bar{y}$ ), such that

(i) $\gamma \Vdash \varphi(0, \bar{b}) \wedge \neg \varphi(a, \bar{b})$,

(ii) $\gamma \Vdash \forall x(\varphi(x, \bar{b}) \rightarrow \varphi(x+1, \bar{b}))$.

By $\mathcal{K} \Vdash W \neg \neg l \neg \Pi_{1}$, we get $\gamma \Vdash \neg \neg \exists x(\neg \varphi(x, \bar{b}) \wedge \forall z<x \varphi(z, \bar{b}))$. Therefore, for some $\delta \geq \gamma$ and some (necessarily nonzero) $d \in M_{\delta}, \delta \Vdash \neg \varphi(d, \bar{b}) \wedge \forall z<d \varphi(z, \bar{b})$. This is a contradiction to the fact that $\gamma$ (and therefore, $\delta$ ) forces $\forall x(\varphi(x, \bar{b}) \rightarrow \varphi(x+1, \bar{b})$ ).

Proposition 1.3 $W \neg \neg l \Pi_{1} \vdash i \neg \Pi_{1}$.

Proof Let $\alpha$ be a node of a Kripke model $\mathcal{K} \Vdash W \neg \neg l \Pi_{1}, \varphi(x, \bar{y})$ negation of a $\Pi_{1^{-}}$ formula, and $\bar{a} \in M_{\alpha}$ of the same arity as $\bar{y}$. To prove $\alpha \Vdash I_{x} \varphi(x, \bar{a})$, assume without 
loss of generality that $\alpha \Vdash \varphi(0, \bar{a})$. It is enough to show that for every $\beta \geq \alpha$, there exists $\delta \geq \beta$ such that, $M_{\delta} \Vdash I_{x} \varphi(x, \bar{a})$, since $\neg \neg I_{x} \varphi(x, \bar{a}) \vdash I_{x} \varphi(x, \bar{a})$. Fix $\beta \geq \alpha$. If $\beta \Vdash \forall x \varphi(x, \bar{a})$, then we may take $\delta=\beta$. Otherwise, by $\beta \Vdash W \neg \neg l \Pi_{1}$, there will exist $\gamma \geq \beta$ such that, for some non-zero $d \in M_{\gamma}, \gamma \Vdash \neg \varphi(d, \bar{a}) \wedge \forall z<d \varphi(z, \bar{a})$. Clearly, such a node $\delta$ has the desired property.

Corollary $1.4 i \Pi_{1} \equiv W \neg \neg l \Pi_{1} \equiv W \neg \neg l \neg \Pi_{1}$.

\section{Forcing and truth}

For a class $\Gamma$ of formulas and a Kripke structure $\mathcal{K}, \Vdash \Leftrightarrow_{\mathcal{K}, \Gamma} \models$ (or just $\Vdash \Leftrightarrow_{\Gamma} \models$ if $\mathcal{K}$ is understood) means that for any node $\alpha$ of $\mathcal{K}$, formula $\varphi(\bar{x}, \bar{y}) \in \Gamma$ and $\bar{a} \in M_{\alpha}$, we have $\alpha \Vdash \varphi(\bar{x}, \bar{a})$ if and only if $M_{\alpha} \models \varphi(\bar{x}, \bar{a})$.

Lemma 2.1 For any Kripke structure $\mathcal{K}$ and any $m \geq 0$, we have:

(i) If $\Vdash \Leftrightarrow_{\Pi_{m}} \models$, then $\Vdash \Leftrightarrow_{\Sigma_{m+1}} \models$.

(ii) If $\Vdash \Leftrightarrow_{\Sigma_{m}} \models$ and $\mathcal{K}$ is a $\Sigma_{m}$-elementary-extension model, then $\mathcal{K} \Vdash P E M_{\Sigma_{m}}$.

(iii) If $\mathcal{K} \Vdash P E M_{\Sigma_{m}}$ is linear, then $\Vdash \Leftrightarrow_{\Pi_{m}} \models$.

Proof (i) and (ii) are straightforward.

(iii) Clearly for any $\mathcal{K} \Vdash P E M_{\Delta_{0}}$, we have $\Vdash \Rightarrow_{\text {Prenex }} \models$. Conversely, assume $\mathcal{K} \Vdash$ $P E M_{\Sigma_{m+1}}$ is linear, $\alpha$ is a node of $\mathcal{K}, \psi(\bar{x}, \bar{a}) \in \Delta_{0}$ and $\alpha \nVdash \forall x_{m+1} \exists x_{m} \cdots Q x_{1} \psi(\bar{x}, \bar{a})$, where $Q \in\{\forall, \exists\}$. Using $P E M_{\Sigma_{m+1}}$, it suffices to show $\alpha \Vdash \neg \neg \exists x_{m+1} \forall x_{m} \cdots Q^{*} x_{1} \neg \psi(\bar{x}, \bar{a})$, where $Q^{*}$ is the quantifier dual to $Q$. If not, there would exist $\beta \geq \alpha$ such that $\beta \Vdash$ $\neg \exists x_{m+1} \forall x_{m} \cdots Q^{*} x_{1} \neg \psi(\bar{x}, \bar{a})$ and so by $P E M_{\Sigma_{m}}, \beta \Vdash \forall x_{m+1} \exists x_{m} \cdots Q x_{1} \psi(\bar{x}, \bar{a})$. By $\alpha \nVdash$ $\forall x_{m+1} \exists x_{m} \cdots Q x_{1} \psi(\bar{x}, \bar{a})$, there exists $\gamma \geq \alpha$ and $c \in M_{\gamma}$ such that $\gamma \nVdash \exists x_{m} \cdots Q x_{1} \psi(\bar{x}, \bar{a})\left[x_{m+1} / c\right]$ and so by $P E M_{\Sigma_{m}}$ again, $\gamma \Vdash \neg \exists x_{m} \cdots Q x_{1} \psi(\bar{x}, \bar{a})\left[x_{m+1} / c\right]$.

But then $\delta=\max \{\beta, \gamma\}$ leads to a contradiction.

Corollary 2.2 Let $\mathcal{K} \Vdash P E M_{\Delta_{0}}$ be linear. Then the following are equivalent:

(i) $\Vdash \Leftrightarrow \Pi_{m} \models$.

(ii) $\mathcal{K}$ is a $\Sigma_{m}$-elementary-extension Kripke model.

(iii) $\mathcal{K} \Vdash P E M_{\Sigma_{m}}$.

It is known that in intuitionistic predicate logic, unlike its classical counterpart, the prenex-normal form theorem does not hold. This is also the case for intuitionistic arithmetic. Indeed, it was proved, by Visser and Wehmeier, that $i P N F$ is $\Pi_{2}$-conservative over $i \Pi_{2}$, were $i P N F$ is the intuitionistic theory axiomatized by $\left(P A^{-}\right)^{i}$ plus the induction scheme restricted to prenex formulas, see [W2, Thm. 3]. However, we have the following:

Corollary 2.3 If $\mathcal{K} \Vdash P E M_{\text {prenex }}$ is linear, then $\mathcal{K} \Vdash P E M$.

For a set $T$ of sentences, $T^{i}$ denotes its intuitionistical closure. In [Bus], the intuition- 
istic theory of the class of $T$-normal Kripke structures is denoted $\mathcal{H}(T)$. Buss axiomatized $\mathcal{H}(T)$ by the universal closures of all formulas of the form $(\neg \theta)^{\varphi}$, where $\theta$ is semipositive (i.e. each implicational subformula of $\theta$ has an atomic antecedent) and $T \vdash_{c} \neg \theta$. It was proved in [M, Cor. 1.2] that, $T^{i} \in \operatorname{range}(\mathcal{H})$ iff $T^{i}=\mathcal{H}(T)$. As a corollary, no fragment of $H A$ extending $i \Pi_{1}$ belongs to the range of $\mathcal{H}$.

Burr's fragments $\Phi_{n}$ of $H A$ are defined as follows, see [Bur2, Sec. 7b]:

(i) $\Phi_{0}=\Delta_{0}$,

(ii) $\Phi_{1}=\Sigma_{1}$,

(iii) For $n \geqslant 2, \Phi_{n}$ consists of all formulas $\forall x(B \rightarrow \exists y C)$, where $B \in \Phi_{n-1}$ and $C \in \Phi_{n-2}$.

Burr showed that these fragments can be considered as normal forms for the formulas of intuitionistic arithmetic. More precisely, he proved:

(i) $I \Pi_{n}=I \Phi_{n}$ for $n \geqslant 0$,

(ii) $\bigcup_{n \in \omega} \Phi_{n}=\operatorname{Form}(L)$ (modulo equivalence in $i \Delta_{0}$ ),

(iii) $I \Pi_{n}$ and $i \Phi_{n}$ prove the same $\Pi_{2}$-formulas for $n \geqslant 0$.

The following was proved by T. Polacik, see [P, lemma 1]:

Fact 2.4 Fix $n \geq 0$. Let $\mathcal{K} \Vdash P E M_{\Delta_{0}}$ be an $\Sigma_{n}$-elementary extension Kripke model. Then, for each $\alpha \in \mathcal{K}$ and each $\varphi \in \Phi_{n}$ we have: $\alpha \Vdash \varphi$ if and only if $M_{\alpha} \models \varphi$.

Proposition 2.5 For each $n \geq 1$, we have $\mathcal{H}\left(I \Pi_{n}\right) \nsubseteq i \Phi_{n}$.

Proof We construct a Kripke structure by putting a model of $I \Pi_{n}$ above a $\Sigma_{n^{-}}$ elementary substructure of it which is not a model of $I \Pi_{n}$, see [HP, P. 222-223] for the existence of such substructures. Using the above fact, it is easy to see that this Kripke model forces $i \Phi_{n}$. So we get a non- $I \Pi_{n}$-normal Kripke model of $i \Phi_{n}$. On the other hand, as it was observed in $[\mathrm{AM}]$ (in the proof of 2.1 (iv)), any theory of the form $\mathcal{H}(T)$ is closed under Friedman's translation and so by [W1], each finite Kripke model of it is $\mathcal{H}(T)^{c}$-normal. So, by [M, lemma 1.2], it must be $T$-normal.

Acknowlegements This research was supported by Institute for Studies in Theoretical Physics and Mathematics (IPM), Tehran, Iran.

\section{References}

[AM] M. Ardeshir and Mojtaba Moniri, Intuitionistic Open Induction and Open Least Number Principle and the Buss Operator, Notre Dame J. Formal Logic 39 (1998), 212-220.

[Bur1] W. Burr, Fragments of Heyting Arithmetic, J. Symbolic Logic 65 (2000), 1223-1240. 
[Bur2] W. Burr, Functionals in Set Theory and Arithmetic, PhD Dissertation, Muenster University, Muenster, 1998.

[Bus] S. Buss, Intuitionistic Validity in T-normal Kripke Structures, Ann. Pure Appl. Logic 59 (1993), 159-173.

[HP] P. Hajek and P. Pudlak, Metamathematics of First-order Arithmetic, SpringerVerlag, Berlin, 1993.

[MM] Morteza Moniri and Mojtaba Moniri, Some Weak Fragments of $H A$ and Certain Closure Properties, J. Symbolic Logic, to appear.

[M] Morteza Moniri, $\mathcal{H}$-theories, Fragments of $H A$ and PA-normality, Arch. Math. Logic, to appear.

[P] T. Polacik, Partially-Elementary Extension Kripke Models and Burr's Hierachy, Bull. Sec. Logic Univ. Lodz 28 (1999), 207-214.

[TD] A.S. Troelstra and D. van Dalen, Constructivism in Mathematics, vol. 1, NorthHolland, Amsterdam, 1988.

[W1] K.F. Wehmeier, Classical and Intuitionistic Models of Arithmetic, Notre Dame J. Formal Logic 37 (1996), 452-461.

[W2] K.F. Wehmeier, Fragments of $H A$ Based on $\Sigma_{1}$-Induction, Arch. Math. Logic 37 (1997), 37-49. 